\title{
Cuerpo y política en jóvenes del movimiento estudiantil universitario (Universidad del Cauca, Colombia)
}

\author{
Willian Andrés Mauna-Rivera, Mg. \\ Universidad del Cauca, Colombia*
}

Gerardo Hernán Jiménez-López, Mg.

Universidad del Cauca, Colombia**

\section{Eduardo Lautaro Galak, Ph.D.}

Universidad Nacional de la Plata, Argentina***

\section{Resumen (analítico)}

Este artículo analiza cómo se configura el cuerpo de jóvenes que participan en dos grupos del movimiento estudiantil de la Universidad del Cauca. Metodológicamente se ubica en el principio de complementariedad, recurriendo a grupos de discusión e historias de vida que provocaron las narrativas. Los resultados destacan que las y los jóvenes configuran colectivamente en y con el cuerpo signos que se (re)producen en las prácticas políticas. Primero, los rituales de iniciación en la práctica política presentan los ritos de paso que introducen al joven en un nuevo campo. Segundo, el cuerpo de la acción, la procesualidad en la práctica política explica el cuerpo que va siendo. Y, tercero, la estética de la práctica política, el cuerpo que manifiesta supone la configuración de un cuerpo para la política, con los atributos para localizarse en este campo.

\section{Palabras clave}

Cuerpo, configuración, juventud, política, organización juvenil, movimiento estudiantil.

\section{Thesauro}

Tesauro de Ciencias Sociales de la Unesco.

\section{Para citar este artículo}

Mauna-Rivera, W. A., Jiménez-López, G. H., \& Galak, E. L. (2020). Cuerpo y política en jóvenes del movimiento estudiantil universitario (Universidad del Cauca, Colombia). Revista Latinoamericana de Ciencias Sociales, Niñez y Juventud, 18(1), 1-20. http://dx.doi.org/10.11600/1692715x.18102

\section{Historial}

Recibido: 22.10 .2018

Aceptado: 05.02.2019

Publicado: 04.10.2019

\section{Información artículo}

Este artículo se desprende de la investigación titulada «Configuración del cuerpo en los grupos que hacen parte del movimiento estudiantil de la Universidad del Cauca» (Popayán, Colombia). Esta fue realizada con financiación aprobada por la Resolución N² 239 del 28 de febrero de 2017, expedida por el Consejo de la Facultad de Ciencias Naturales, Exactas y de la Educación de la Universidad del Cauca, entre el 07 de agosto de 2015 y el 02 de marzo de 2018. El artículo es una revisión de esa investigación por parte de los tres autores que la firman. Área: Ciencias Sociales; subárea: Sociología. 


\section{Body and policies in young university student movement (Unicauca, Colombia)}

Abstract (analytical)

This paper analyzes the configuration of the bodies of young people who participate in two student political movements at the Universidad del Cauca (Colombia). Methodologically, the study is based on the principle of complementary actions, using discussion groups and life stories to build narratives. The results show how the young people collectively configure, both in and with the body, signs that are (re)produced in their political practices. First, the initiation rituals in political practice present the rites of passage that introduce the young people to this new area. Second, the actions of their body are analysed. The procedurality of their political practices explain the body that is being. Third, in the aesthetics of political practice, the body implies the configuration of a body for politics with the necessary attributes to act in this field.

Keywords

Body, configuration, youth, politics, youth organizations, student movements.

\section{Corpo e política em jovens do movimento universitário estudantil (Unicauca, Colômbia)}

Resumo (analítico)

Este artigo analisa como se configura o corpo de jovens que são parte de dois grupos do movimento estudantil da Universidad del Cauca (Colômbia). Metodologicamente o texto se localiza no princípio de complementariedade, recorrendo aos grupos de discussão e as histórias de vida que geraram as narrativas. Os resultados salientam que as e os jovens configuram coletivamente em e com o corpo sinais que se reproduzem nas práticas políticas. Primeiro, os rituais de iniciação na prática política presentam os rituais de passagem que introduzem ao jovens em um novo campo. Segundo, o corpo da ação, a processualidade na prática política explica o corpo que vai surgindo. E terceiro, a estética da prática política, o corpo que manifesta supõe a configuração de um corpo para a política, com os atributos para localizar-se neste campo.

Palavras-chave

Corpo, configuração, juventude, política, organização juvenil, movimento estudantil.

Información autores

[*] Licenciado en Educación Básica con énfasis en Educación Física, Recreación y Deportes de la Universidad del Cauca. Magíster en Educación, Estudios del Cuerpo y la Motricidad de esta misma institución universitaria. Investigador del Grupo de Investigación Urdimbre, y gestor de cultura y bienestar de la Universidad del Cauca. (iD) 0000-0002-2112-2045. Índice H5: 1. Correo electrónico: wmauna@unicauca.edu.co

[**] Licenciado en Educación Básica con énfasis en Educación Física, Recreación y Deportes de la Universidad del Cauca. Magíster en Educación, línea Saber Pedagógico, de esta misma institución universitaria. Candidato a doctor en Ciencias de la Educación de la Universidad del Cauca-RudeColombia. Profesor de la Universidad del Cauca. ID 0000-0002-1302-1711. Índice H5: 1. Correo electrónico: gjimenez@unicauca.edu.co [***] Profesor en Educación Física de la Universidad Nacional de la Plata, Argentina (UNLP). Magíster en Educación Corporal y doctor en Ciencias Sociales de esta misma institución universitaria. Post-doctor en Educação, Conhecimento e Integração Social de la UFMG. Investigador del Conicet y profesor de la UNLP. (iD) 0000-0002-0684-121X. Índice H5: 8. Correo electrónico: eduardogalak@gmail.com 


\section{Introducción}

Este artículo pretende mostrar analíticamente las implicaciones que las prácticas políticas tienen en el cuerpo y viceversa, ya que las y los jóvenes históricamente han sido comprendidos como sujetos relativamente neutrales ante los procesos sociales y políticos. Como plantea Reguillo (2013), en la contemporaneidad se los ha concebido como agentes importantes en la constitución de la sociedad, pero exclusivamente bajo las condiciones que los adultos les imponen desde las instituciones y las actuales formas de consumo, administradas a partir de un sistema cada vez más especializado de vigilancia y control social-jurídico que funciona como un aparato de contención y sanción a sus prácticas.

Un primer punto a señalar es que ello puede deberse a que la política está fuertemente arraigada a la construcción moderna de ciudadanía, y que la condición de ciudadano se adquiere en el pasaje de la juventud a la adultez (resumida en cumplir una determinada edad, generalmente los 18 años). Una segunda causa está relacionada con la moratoria social que presenta lo juvenil como etapa de transición, en la cual las y los jóvenes deben adquirir todas las habilidades, conocimientos y valores para integrarse al mundo adulto. Galak (2012) propone en esta perspectiva que la etapa juvenil es una mixtura entre cierta madurez biológica y una inexperiencia social, esto es, «una instancia de transición a la vida adulta» (p. 29). En correspondencia a lo anterior, Font (2015) plantea que esta visión, en lo que se refiere a las relaciones de poder, mantiene a los jóvenes al margen de la participación activa que se internaliza en su subjetividad.

Por su parte, y en el contexto específico de la ciudad de Popayán, se constituyen dos imaginarios que conciben al joven como pasivo: en primer lugar está el de ser denominada la «ciudad blanca» (planteado por Buendía, 2014); ello muestra cómo institucionalmente se estructuran sentidos conservadores de pureza y religiosidad, lo cual restringe las prácticas juveniles que subvierten este orden; y, en segundo lugar, está el imaginario de la moratoria social, ratificado en el hecho de que, al ser Popayán una ciudad universitaria, se entiende que las y los jóvenes están dedicados a instruirse en las universidades para luego insertarse en la vida laboral adulta. 
$\mathrm{Al}$ contrario de esta postura, la investigación muestra que las y los jóvenes han creado espacios alternos a los que les brinda la institucionalidad, configurando grupalidades alrededor de diversas prácticas que les permiten participar como sujetos políticos en la construcción de sociedad. Tal es el caso de los dos grupos estudiados, los cuales hacen parte del movimiento estudiantil de la Universidad del Cauca.

En consecuencia, este trabajo puso la mirada sobre las prácticas políticas que realizan las y los jóvenes en el marco de la movilización estudiantil. Prácticas que, siguiendo los argumentos de Bourdieu (2007), son generadas como sistemas de pensamiento que condicionan las conductas y los sentidos comunes, permitiéndole a los sujetos desenvolverse en campos específicos de las grupalidades políticas.

En el caso de los grupos estudiados, y siguiendo los planteamientos de Butler (2017), se entiende que las prácticas políticas «generalmente responden a objetivos políticos de carácter distinto, pero en todas sucede algo similar: los cuerpos se reúnen, se mueven y hablan entre ellos, y juntos reclaman un determinado espacio como espacio público» (p. 75). Esto implica una relación directa con los cuerpos que se reúnen en las calles, las plazas y otros espacios públicos y privados a desarrollar acciones para aparecer como agentes, reclamando «una reivindicación corporeizada de una vida más vivible» (p. 31). Lo anterior sitúa al cuerpo como primer territorio de resistencia, desplegando técnicas que atraviesan el espacio urbano (Diz, 2018).

Como resultado de lo expuesto, surgen una serie de interrogantes, tales como: ¿qué papel juega el cuerpo en los escenarios de la práctica política?, ¿cómo estas prácticas políticas se inscriben en el cuerpo?, ¿cómo se manifiesta lo corporal en las prácticas políticas de los colectivos estudiados? Se parte de entender que, en sus prácticas, las y los jóvenes subvierten los usos del cuerpo y rompen con los esquemas establecidos por la sociedad en el plano de lo estético y lo político, agrupándose en la defensa de sus derechos y reclamando un reconocimiento por parte de la universidad y de la sociedad. En este sentido, las reflexiones alrededor del tema giran en torno a cómo las prácticas políticas se apropian de sus cuerpos como primer escenario de protesta (Pabón \& Hurtado, 2016), para mostrar sus diferencias y singularidades respecto al resto de la sociedad. De esta manera, cobran un nuevo significado los planteamientos esbozados por Bourdieu (1994) en referencia a la relación cuerpo y sociedad, en tanto que el primero funciona como un lenguaje mudo de lo segundo (Galak, 2010), que es incorporado en procesos históricos sucesivos: efecto de la «historia hecha cuerpo», los habitus se manifiestan en prácticas que tienen por objeto lo corporal y, entonces, el cuerpo es el producto social en donde se 
inscriben las prácticas. En el caso particular estudiado, esto se observa en la disputa entre la reproducción que implican los procesos de educación y las rupturas al orden político construido históricamente que los agentes despliegan en sus prácticas.

En este contexto, el cuerpo, en tanto capital, cobra una significación teórica para pensar las prácticas políticas, especialmente desde las ciencias sociales, que en los últimos años han desarrollado una serie de posicionamientos al respecto. Entre otras, son significativas las conceptualizaciones del cuerpo como técnicas corporales de Mauss (1979), la reproductibilidad de lo social mediante la incorporación que plantea Bourdieu (1994) y la materialización concebida por Butler (2002). En este orden de ideas, este trabajo pretende poner como centro de análisis al cuerpo de las y los jóvenes, para analizar cómo este se configura a través de prácticas políticas, tema que ha sido poco explorado en este registro y que es fundamental en la comprensión de la sociedad contemporánea.

Para llevar a cabo esta indagación se revisaron algunos trabajos investigativos sobre la tríada cuerpo, jóvenes y práctica política, dividiéndolos en dos tendencias temáticas, en absoluto antitéticas entre sí: una primera referida a las prácticas de participación juvenil, prácticas políticas y movimientos sociales; en ella se encontraron estudios que, generalmente desde una perspectiva etnográfica, abordan cómo se configuran las militancias juveniles políticas, su participación en contextos sociales, los ordenamientos al interior de esos grupos y cómo se articulan con otros; muestran la forma de incorporación de nuevas disposiciones sociales y políticas en las y los sujetos jóvenes, que les permiten participar en el contexto en el cual se desenvuelven desde diferentes instancias (Alvarado et al. 2012; Dukuen, 2018; Giraldo \& Ruiz, 2019; Patiño et al., 2014). La segunda tendencia temática presenta específicamente tópicos referidos a los cuerpos juveniles, las subjetividades y la acción política (Díaz \& Alvarado, 2012; Figueroa-Grenett, 2018; Hurtado, 2011; Pabón \& Hurtado, 2016). Se incluyen en esta dirección algunos trabajos desarrollados en torno a la forma en que las y los jóvenes construyen subjetividades a partir de prácticas políticas y culturales que se constituyen como prácticas de subjetivación; estos disponen sus cuerpos con nuevos significados en torno a lo social/político, generalmente en espacios no institucionalizados, en donde se configura un sujeto joven con creativas formas de acción política. En ellas el cuerpo está presente a través de sus técnicas y usos como una «ciudadanía en acto».

Estas reflexiones sobre la problemática y los antecedentes permitieron establecer, como objetivo del trabajo, el comprender cómo se configura el cuerpo de las y los jóvenes adscritos a dos grupalidades políticas estudiantiles. Para ello se buscó: 1) interpretar 
las transformaciones corporales que tienen los y las jóvenes al interior de los grupos de las instancias políticas universitarias; 2) reconocer el papel que juega el cuerpo en los escenarios de la práctica política; y 3 ) poder describir las prácticas en las cuales el cuerpo es específicamente un espacio de intervención o manifestación política.

Se proponen como centro de atención las formas creativas de participación en la esfera pública de las y los jóvenes, y cómo estos van incorporando las significaciones simbólicas construidas por los grupos a los que se vinculan; es decir, aquellas prácticas, saberes y discursos que se constituyen como una «necesidad social vuelta naturaleza, convertida en esquemas motrices y automatismos corporales» (Bourdieu, 2007, p. 111), entendiendo los procesos por los cuales lo social se objetiva en el cuerpo; esto es, comprender cómo lo social está en el cuerpo y el cuerpo en lo social, objetivándose en técnicas y usos (Galak, 2010). Así, esta perspectiva pretende interpretar cómo las y los jóvenes re-significan, re-construyen y re-producen las formas tradicionales de ser y hacer en el campo de la política, para entender cómo mediante sus prácticas procuran romper con las estructuras dominantes establecidas y ponen el cuerpo como primer territorio de poder, en el cual se establece la resistencia y la desobediencia.

De tal manera, el cuerpo se constituye como potencia limitada ${ }^{1}$ de nuevas formas de la práctica política que configura renovadas disposiciones sociales. En este sentido, se comprende que lo corporal, en los escenarios de la práctica política estudiantil, se instituye como resistencia ante los usos establecidos que lo alienan; de allí que se parta de entender que poseen la capacidad de hacer historia. Como dice Bourdieu (2007), los agentes pueden crear pensamientos, percepciones y acciones de manera condicional y condicionada por los límites que les impone la producción histórica. Es una tensión permanente entre las estructuras dominantes de la sociedad y lo que se está proponiendo desde los intersticios de las prácticas juveniles.

La intención es desarrollar una reflexión que contribuya a reconocer en las y los jóvenes sus potencialidades para generar procesos de participación política en la universidad y, a través de ello, en la sociedad en general, vinculándose políticamente en prácticas que producen cuerpos singulares y generan una nueva posibilidad de comprender la relación cuerpo-política-sociedad.

\footnotetext{
${ }^{1}$ En «Esbozos de una teoría de la práctica de educar: Pierre Bourdieu, educación de los cuerpos, violencia y capital simbólico» (Galak, 2015) se afirma que lo social incorporado se constituye para la sociología bourdieuana en forma de habitus que tienden a reproducirse en prácticas corporales como potencias limitadas.
} 


\section{Método}

Este artículo presenta parte de los resultados de una investigación cualitativa realizada entre los años 2015 y 2018. Su despliegue metodológico está amparado desde el principio de complementariedad, propuesto por Murcia y Jaramillo (citados por Hurtado, 2011), que permitió articular en su diseño diferentes técnicas investigativas en la recolección de la información y la comprensión de las realidades sociales/corporales desde las experiencias de jóvenes activistas de la Universidad del Cauca. ${ }^{2}$ Desde esta perspectiva, las metodologías a las que se recurrió fueron la observación participante, los grupos de discusión y las historias de vida.

Frente a lo anterior es necesario precisar, desde las conceptualizaciones de Galeano (2004), que: 1) la observación participante consiste en observar y registrar las interacciones, comportamientos, ritmos y cotidianidades de los colectivos estudiados; 2) los grupos de discusión son una estrategia de investigación interactiva grupal que busca «investigar formas de conducta, representaciones sociales y simbólicas, y discursos ideológicos asociados al objeto de estudio» (p. 19o); y 3 ) las historias de vida pretenden «generar visiones alternativas de la realidad social mediante la reconstrucción de vivencias personales» (p. 6o).

A partir de este proceso obtuvimos un banco de información que se categorizó ${ }^{3}$ con teoría fundamentada (Strauss \& Corbin, 2002) y que permitió, por un lado, sintetizar la información a través de agrupaciones de datos y, por el otro, relacionar con la teoría formal.

Los resultados se construyeron a partir de las narrativas obtenidas en los grupos de discusión y las historias de vida; con ello se logró reflexionar sobre las y los jóvenes en relación con sus prácticas políticas a la luz de la teoría formal, lo cual permitió ampliar los entramados de sentido sobre la configuración del cuerpo político de los jóvenes adscritos al movimiento estudiantil.

\footnotetext{
2 La Universidad del Cauca es una institución de educación superior de carácter público, ubicada en la ciudad de Popayán (Cauca, Colombia). En el segundo semestre de 2018 se matricularon 16155 estudiantes en la modalidad de pregrado (Universidad del Cauca, 2019).

3 En esta perspectiva, categorizar es un «proceso no matemático de interpretación, realizado con el propósito de descubrir conceptos y relaciones en los datos brutos y luego organizarlos en un esquema explicativo» (Strauss \& Corbin, 2002, p. 12).
} 


\section{Sobre los participantes y el diseño metodológico}

Ahora bien, los participantes de la investigación fueron jóvenes universitarios pertenecientes a dos colectivos: Federación Colombiana de Estudiantes (Cauca) y Organización Estudiantil Territorio Libre, los cuales hacen parte de los diversos grupos que conforman el movimiento estudiantil. Las y los jóvenes que integran estas colectividades se distinguen por aunarse alrededor de la defensa de la educación superior pública. Son personas que se caracterizan, según Hurtado (2011), por disponer de liderazgo y compromiso social; les gusta reflexionar y debatir críticamente la situación económica, política y social que atraviesa tanto la universidad como el país. Los grupos están conformados por jóvenes que en su mayoría son estudiantes de los 46 programas de pregrado que ofrece la universidad y se reúnen en espacios que la institución ha dispuesto para ello (oficinas o salones).

En cuanto al diseño metodológico, este trabajo propuso tres momentos de desarrollo, siguiendo los planteamientos de Galeano (2004) y el principio de complementariedad (Murcia \& Jaramillo, citados por Hurtado, 2011). Estos momentos son: 1) la exploración, que consistió en un acercamiento inicial a las y los jóvenes pertenecientes a diversos grupos que conforman el movimiento estudiantil, a través de la observación participante; esto se registró en ocho diarios de campo que permitieron seleccionar a los dos colectivos que harían parte de la investigación y mostrar los tópicos generales de las prácticas políticas. 2) La focalización a través de cuatro grupos de discusión ${ }^{4}$ (dos por grupo), en los que participaron no menos de ocho estudiantes con los dos colectivos elegidos en el primer momento; este escenario permitió ampliar la comprensión sobre las prácticas grupales y generar líneas para configurar el tercer momento. 3) La profundización, momento en el cual se realizaron dos historias de vida temáticas 5 que habilitaron sentidos de la práctica política a nivel personal. Dicho ejercicio se desarrolló a través de entrevistas

\footnotetext{
4 Los grupos de discusión se orientaron según tópicos emergentes de las observaciones en el primer momento de exploración; estos son: 1) el espacio institucional como espacio de la práctica política juvenil; 2) el encuentro en la práctica política: la configuración de un espacio de interacción y proximidad; 3 ) la politización del cuerpo en la práctica política; y 4) estar en la lucha: procesos de movilización política no violenta. Con esto se realizó una guía de zo preguntas orientadoras, desarrolladas en dos sesiones por cada grupo y grabadas en audio para luego ser transcritas y analizadas.
}

5 Estas hacen referencia a aquellas que indagan sobre hechos o procesos particulares en la vida de un sujeto (Galeano, 2004). 
biográficas abiertas ${ }^{6} \mathrm{y}$ a profundidad, entrevistando a un joven de cada grupo que tuviera una trayectoria significativa en su colectividad. En esta investigación se optó por utilizar narrativas surgidas de los grupos de discusión y de las historias de vida, lo que generó un amplio panorama de las prácticas políticas a nivel grupal y personal, las cuales fueron sintetizadas a través de aportes de la teoría fundamentada.

\section{Resultados El cuerpo: su re-significación en la práctica política}

A continuación se despliegan una serie de consideraciones acerca de los resultados de la investigación desarrollada. En principio, vale la afirmación de que el cuerpo, como configuración política, evidencia en primer lugar los rituales de entrada al grupo de los participantes en colectivos políticos estudiantiles. En segunda medida, se expresa cómo, a partir del activismo político en el movimiento estudiantil, comienza un proceso de incorporación de la práctica, en tanto con el cuerpo (la presencia, la materialidad) y en el cuerpo (la encarnación de lo social, lo instituyente y normalizado). La intención de fondo es pensar los sentidos del cuerpo en las políticas y las políticas en los cuerpos, mientras que se estudia «el cuerpo que manifiesta» activamente en los estudiantes de la Universidad del Cauca.

\section{El ritual de iniciación en la práctica política juvenil}

El ritual de iniciación hace referencia al pasaje o tránsito de un estado social a otro. Reguillo (2013) lo considera como un proceso de «tránsito hacia una nueva situación y les confiere a los participantes iniciados los atributos de su nueva posición» (p. 100). Las y los jóvenes expresan en los relatos que antes de tener contacto con las prácticas del movimiento estudiantil, aunque habían asistido a algunas acciones, estas no habían tenido incidencia en ellos hasta que surgió un interés por las mismas. Vale la pena hacer notar que el interés surge en escenarios de la práctica política (jornadas, marchas, plantones, entre otros).

\footnotetext{
6 Las entrevistas se realizaron sobre tres tópicos emergentes del preanálisis de los grupos de discusión: 1) el cuerpo: sus transformaciones y formas en la práctica política; 2) cuerpos, acciones y discursos; y 3) el no lugar de los cuerpos. Con estos se logró formular un total de 27 preguntas que pretendían generar narrativas sobre la vida de cada joven del movimiento estudiantil, las cuales fueron grabadas en audio y luego transcritas para su posterior análisis comprensivo.
} 
Antes de iniciar nunca había tenido experiencias de movilización; había salido a marchas y estas cosas, pero nunca el tema de organizarme me había interesado. Durante el paro de 2013, hay una invitación de un compañero, durante una jornada de carteles, a una marcha de antorchas que terminó en el parque Caldas. Ahí fue donde realmente hubo la invitación a esta oficina de la FEU [Federación de Estudiantes Universitarios]. (ME/GD2/R6) ${ }^{7}$

Esto es, como dice Bourdieu (2007), entrar al «mundo práctico que se constituye en la relación con el habitus como sistema de estructuras cognitivas y motivadoras, es un mundo de fines ya realizados, modos de empleo o pronunciamientos por seguir, y de objetos dotados de un carácter teleológico permanente» (p. 87). Esta propuesta presenta que las y los jóvenes movilizados configuran esquemas o estructuras que les permiten hacer, pensar y sentir en las prácticas políticas. Además, ellas son estructurantes, ya que al mismo tiempo producen percepciones, pensamientos y acciones que hacen posible plantear que las prácticas son aprendidas y aprehendidas por el cuerpo, mediante un proceso de familiarización que inicia en relación con los simbolismos que distintos estudiantes ya han incorporado en sus grupos. En palabras de uno de los estudiantes:

Yo empecé desde secundaria. La forma como entré fue en un grupo de estudiantes de una organización que llegaron a mi colegio, hablaron del libre desarrollo de la personalidad; entonces, en esa época uno está en secundaria y uno quiere piercing, quiere el pelo largo, quiere el pantalón entubado; cosas propias de la juventud que yo comencé a hacer. (ME/ $\left.\mathrm{GD}_{2} / \mathrm{R}_{1}\right)$

En el caso anterior es posible postular que el joven es persuadido por los discursos incorporados que circulan en el mencionado grupo, los cuales lo interpelan a actuar sobre sus formas de llevar el cuerpo, orientado por esquemas de percepción, reproducidos en la organización, que permiten a los sujetos habitar el nuevo espacio social (Bourdieu, 2007). Puede argumentarse, entonces, que el cuerpo es constituido en la articulación de una doble historia: la de su trayecto individual y la del colectivo en el que participa. Por eso cuando las y los jóvenes entran a una grupalidad del movimiento estudiantil, dotado de significaciones simbólicas, se inicia un proceso particular de construcción del cuerpo.

\footnotetext{
7 Es preciso aclarar que se pautó con los entrevistados un anonimato en la publicación de sus respuestas. De allí que se utilice un código alfanumérico que sirve para ubicar la procedencia del relato. Por ejemplo, en ME/GD2/ R1, ME corresponde a Movimiento Estudiantil; GD2 a la técnica de recolección utilizada (grupo de discusión) y R1 corresponde al número del relato o segmento nominado (relato 1).
} 
De lo anterior se puede deducir que la eficacia simbólica de los colectivos políticos juveniles «podría encontrarse en el poder que otorga sobre los otros, y especialmente sobre sus cuerpos y sus creencias» (Bourdieu, 2007, p. 112). La entrada a un nuevo campo de prácticas políticas es denominada como ritual, ya que través de acciones simbólicas se les permite a las y los jóvenes empezar a comprender nuevas estructuras de significados propias de los grupos estudiantiles.

\section{El cuerpo de la acción: procesualidad en su configuración}

Otro tópico de reflexión es el proceso para ir constituyendo un cuerpo para la política. Se pretende mostrar la relación prácticas políticas-cuerpo como generadora de procesos de incorporación de los signos del grupo que se instalan procesualmente en el sujeto de forma permanente y que permiten construir estructuras de percepción que vinculan a las y los jóvenes en el campo específico de las grupalidades estudiadas. Sobre este tema, Le Breton (2002) plantea que «las representaciones sociales le asignan al cuerpo una posición determinada dentro del simbolismo general de una sociedad» (p. 13). En este caso, se trata de un grupo que ha estructurado históricamente disposiciones corporales relacionadas con las prácticas apolíticas puestas en cuestión. Disposiciones que se adquieren en el devenir de las y los jóvenes en las prácticas (no necesariamente políticas, pero si en relación con ellas) a través de nuevos simbolismos que descubren en diversos escenarios. El siguiente testimonio es una muestra de ello:

Bueno, pues yo estudié en un colegio católico y desde noveno empecé con un grupo de amigos a escuchar punk. Mi hermana está en la universidad y tocaba bajo, entonces nos montamos una banda de punk. Fue algo que me ayudó a incentivar un poco el tema de la lucha social y la lucha por el bienestar de las mayorías, porque las letras del punk, que si bien son muy agresivas, son letras escritas sobre la realidad del momento. Empecé a estudiar las letras y el lenguaje musical, el estilo de vida y la contracultura; ese fue mi recorrido, más que todo en el colegio. Cuando entro a la universidad, trato de desarrollar un poco el tema musical, pero finalmente me dedico al tema del liderazgo estudiantil y de formación al interior de la organización. (RV/E1/r1)

Este ejemplo muestra el proceso por el cual se van incorporando estructuras de sentido desde procesos específicos como la música, en espacios que ellos y ellas han constituido para sus prácticas y que son alternos a los que les ofrece la institucionalidad de la educación (como la escuela). Desde la perspectiva bourdieuana se posiciona que las y los jóvenes, que ya cuentan con disposiciones permanentes de estructuras sociales dominantes, 
incursionan en otros campos dotados de agentes, creencias y capitales simbólicos que los ponen frente a nuevas formas de «estar, de hablar, de caminar, y, por ende, de sentir y de pensar» (Bourdieu, 2007, p. 113).

En el siguiente testimonio, un joven habla sobre sus mudanzas cuando entra en el colectivo estudiantil: «Cambio de mentalidad como de muchas necesidades que a uno le parecen no tan importantes cuando estaba más joven: ropa cara, zapatos caros; ese tipo de gustos se perdieron, porque me di cuenta que no eran realmente importantes» (ME/ $\left.\mathrm{GD}_{2} / \mathrm{R}_{5} \mathrm{O}\right)$.

Los procesos que las y los jóvenes inician en los colectivos en que se introducen desplazan las formas sociales establecidas y legitimadas de llevar el cuerpo, interpelando su posición en oposición. Los agentes descubren otras posibilidades corporales que engendran representaciones para el juego en la práctica política; «el cuerpo cree en aquello a lo que juega» (Bourdieu, 2007, p. 118).

Una vez integrados al grupo, los militantes incorporan significantes de la política, principalmente a través de acciones y discursos, empezando a desafiar la forma dominante de reproducción de los cuerpos. La transformación se adquiere en el devenir de la práctica; las representaciones son asignadas en una permanente relación del sujeto con el grupo, en la cual se irá constituyendo un cuerpo para la política; como dice Díaz (2012), «es un cuerpo que va siendo» (p. 117). Un ejemplo de ello lo entrega el siguiente relato: «También el uso de las botas. A mí no gustaban las botas antes, me parecían algo muy incómodo, muy pesadas para llevar. Estando acá, en la organización, comencé a ponerme mis botas y siempre ando con ellas» (ME/GD2/R43).

Siguiendo los planteamientos de Díaz (2012), se puede argumentar que:

El cuerpo se hace en relación con otros, el cuerpo es significado, es nominado, denominado con diferentes atributos, se le representa, ya no como el cuerpo en sí, sino como el cuerpo para el otro, el cuerpo para los otros, por lo que se asume como cuerpo simbólico. (p. 118)

Y es justamente esta dimensión relacional la que permite ir adquiriendo las formas de ser cuerpo que los jóvenes han construido en la historia de su organización. Este registro supone las transformaciones subjetivas de las y los jóvenes ya socializados a un nuevo campo, con nuevos agentes, creencias, capitales y significados, los cuales empiezan a recorrer al cuerpo con nuevas formas de poder que resisten a los dispositivos más opresivos que pretenden alienarlos. Es como una socialización secundaria que, según Berger y 
Luckmann (1968), «requiere la adquisición de vocabularios específicos de "roles", lo que significa por lo pronto la internalización de campos semánticos que estructuran interpretaciones y comportamientos de rutinas dentro de un área institucional» (p. 175).

\section{La estética de la práctica política: «el cuerpo que manifiesta»}

La propuesta central de este artículo es reflexionar acerca del cuerpo como manifestación política de las y los jóvenes agrupados en colectivos que conforman el movimiento estudiantil, presentando de manera sustancial las significaciones y re-significaciones que estas personas le asignan para disponerlo como un cuerpo para la política. Se trata de mirar todas las formas instituyentes de relacionarse consigo mismo, con los otros y con el espacio dentro del contexto de militancia política. Se habla entonces de un cuerpo con nuevas sensibilidades, nuevos usos y nuevas estéticas. Es, como explica Bourdieu (2007), la configuración de una nueva hexis corporal. ${ }^{8}$ Desde los rituales de iniciación en la práctica, seguido de la procesualidad que los va haciendo cuerpos, surge el cuerpo para la política propia de los colectivos estudiantiles. Desde lo anterior, se puede decir que:

Las piernas, los brazos están llenos de imperativos adormecidos. Y uno no termina nunca de enunciar los valores hechos cuerpo por la transmutación operada por la clandestina persuasión de una pedagogía implícita, capaz de inculcar toda una cosmología, una ética, una metafísica, una política, [...] y de inscribir en los detalles en apariencia más insignificantes del vestir, de la compostura o de las maneras corporales y verbales los principios fundamentales de la arbitrariedad cultural, situados así fuera de la influencia de la conciencia y de la explicación. (Bourdieu, 2007, p. 112)

Las y los jóvenes activistas configuran sus espacios vitales desde propuestas estéticas corporales 9 que revisten el poder que ellos han creado en su organización. Por ende, empiezan a utilizar otro tipo de ropa, a adquirir otros discursos y a realizar otras acciones que son un lenguaje corporal que expresa las ideologías políticas de su activismo. Mediante la

8 Para Bourdieu (2007), las hexis corporales se presentan en relación con los habitus, refiriéndose a las formas de llevar el cuerpo, a las posturas y disposiciones permanentes de los significados incorporados al mismo. Esto es, formas permanentes de hablar, caminar y ser del cuerpo; maneras de sentir y de pensar. Para un mayor desarrollo de este tema se recomienda la lectura de «El concepto cuerpo en Pierre Bourdieu: un análisis de sus usos, sus límites y sus potencialidades» (Galak, 2010).

9 La estética corporal hace referencia a la forma como los jóvenes exteriorizan lo que incorporan, lo que implica mostrarlos como agentes capaces de actuar sobre sí mismos. Pabón y Hurtado (2016) mencionan que las estéticas corporales son la expresión de la interioridad en las cuales los jóvenes se apropian de sus cuerpos como «territorios simbólicos». 
ropa, las botas, la mochila, la risa, el cabello o el tatuaje, desdibujan las formas establecidas que producen al cuerpo y manifiestan su descontento ante los sistemas que los alienan. Con respecto a esto, un joven plantea:

Principalmente en el colectivo cambio mi manera de vestir. Entonces, cuando yo entré a la organización, pues ya miro camisas del Che, de Marcha Patriótica o ya me coloco camisas hasta de la misma Federación; entonces uno va cambiando esa manera de vestir. ¿Me hago entender? (ME/GD2/R53)

El joven muestra cómo estar en la colectividad requiere de un cuerpo dotado de signos ya construidos per se. Es decir, que con la vestimenta se reconoce como parte del grupo y se posiciona como sujeto de la práctica. «La ropa cumple [entonces] un papel central para reconocer a los iguales y distanciarse de los otros; tiene una potencia simbólica capaz de establecer diferencias entre los cuerpos juveniles, sobre los cuales una mirada superficial podría leer homogeneidad» (Reguillo, 2013, p. 69).

En los colectivos políticos juveniles, sus integrantes, a través de sus discursos y acciones, dotan de significado a los elementos que portan, como la ropa, la bandera y otros objetos que se convierten en emblemáticos de su práctica materializada en una estética corporal, la cual se sitúa en el campo de la política. Ellos lo exteriorizan mediante símbolos con un valor discursivo que trasciende su forma y su función.

Entonces, cuando ya llego a mi casa, y me veían con la camisa de Marcha Patriótica o con la camisa del Che, o una camisa de una organización, empezaban a preguntar: ¿Bueno, hijo, y esto qué? Y uno contesta: Mira mamá que esta es la camisa de Marcha Patriótica, de una organización social. (ME/GD2/R54)

En este sentido, las y los jóvenes atacan líneas del poder dominante en el campo político, a través de una estética de los cuerpos fuera de los patrones de normalidad que pretende descolectivizarlos, ya que en sus significados manifiestan nuevos lenguajes llenos de expresiones carnavalescas del poder, con dispositivos que configuran los cuerpos juveniles que subvierten los órdenes reproducidos en la sociedad, como destacan Pabón y Hurtado (2016), apoyados en Bajtín. En consecuencia, el contenido de la vida interior de los sujetos crea sus propias expresiones de sí mismos y conlleva a la externalización de estéticas de los simbolismos internalizados. Siguiendo esta lógica, los patrones estéticos creados al interior de las colectividades e incorporados en las y los jóvenes juegan un papel muy importante en el reconocimiento entre ellos mismos y los otros. Como lo expo- 
ne Reguillo (2013), existe «la tendencia a distinguirse de otros grupos de pares mediante ciertos usos estéticos y a través de marcas simbólicas» (p. 88).

Se denota así que el cuerpo se convierte en una forma objetiva que externaliza las significaciones políticas del movimiento estudiantil, siendo atravesado por el contenido estético que resisten los sujetos y manifestando una ideología de desconcierto con las entidades que administra el Estado. De esta forma, «el cuerpo puede contener el mensaje, ser soporte del mensaje, o ser él mismo el mensaje, ya que en el cuerpo se produce y articula la ideología política» (Sasson-Levy \& Rapoport, citados por Enguix, 2012, p. 78).

Entonces, el vestir y los usos corporales, las botas, las camisetas negras, los estampados con logos de organizaciones y personajes políticos, la mochila, así como los usos específicos del cuerpo en manifestaciones públicas, entre otros, se incorporan; esto último, en el sentido que hacen parte del sujeto como forma permanente que los diferencia y los distingue, pero que principalmente para ellos se expresa en una forma de manifestación. Con respecto a lo anterior, un joven indica:

Bueno, pues [en] mi caso fue muy fuerte el pertenecer a esto, porque (...) cuando yo entré a secundaria, me acuerdo que fue muy difícil que entrara a un grupo donde me dijeron: usted puede tener el pelo largo. Y ¡ve!, pues me voy a dejar el pelo largo. Y en la casa como que ¡ve!, ¿qué te está pasando? (ME/GD2/R41)

En este orden de ideas, se puede plantear que el cuerpo se reviste de simbolismos ideológicos y políticos que les permiten a las y los actores desarrollar sus prácticas políticas estudiantiles, que se manifiestan en los espacios públicos y cotidianos; es decir, el cuerpo de cada joven se construye entre sus potencias individuales y los lineamientos grupales para posibilitar la participación en diversos escenarios que exige el activismo estudiantil.

\section{Discusión}

En este escrito se expusieron algunos resultados emergentes de una investigación realizada con dos colectivos que hacen parte del movimiento estudiantil de la Universidad del Cauca. En los antecedentes de estudio se encontraron dos líneas de trabajo: por un lado, aquellas indagaciones que se refieren a las juventudes, las prácticas políticas y los movimientos sociales; y, por el otro, las que se dirigen a comprender específicamente 
los cuerpos juveniles y las subjetividades. En este sentido, cabe resaltar que esta investigación coincide en sus principales líneas con los desarrollos teóricos esbozados por Dukuen (2018) quien, desde la perspectiva de Bourdieu, afirma que la socialización política se (re)produce por la incorporación de (dis)posiciones políticas. Es decir, se comparte la idea de que la militancia universitaria configura usos y sentidos corporales particulares en las y los jóvenes, creando espacios en donde se llevan a cabo prácticas que los constituyen como cuerpos para la política. A su vez, esta investigación retoma el argumento de Patiño et al. (2014) y de Alvarado et al. (2012), quienes indagan las incidencias políticas de las y los jóvenes en los contextos en donde intervienen; no obstante, se distancia de estos trabajos al afirmar que las prácticas de militancia interpelan directamente al cuerpo en sus (dis)posiciones, incorporándose en forma de técnicas corporales. Precisamente hacia una argumentación de lo anterior es que se dirigen nuestras siguientes consideraciones finales.

A partir del trabajo de campo investigativo sobre los dos grupos que hacen parte del movimiento estudiantil de la Universidad del Cauca se construyeron una serie de reflexiones para responder a la siguiente pregunta: ¿cómo se configura el cuerpo de las y los jóvenes en los grupos que hacen parte del movimiento estudiantil? En principio, e incluso reconociendo que esto no implica necesariamente una estricta novedad, cabe resaltar que la indagación muestra que lo corporal es interpelado por una diversidad de significaciones políticas que son constitutivas de las prácticas políticas de militancia. En efecto, como primer resultado, puede verse cómo estas significaciones se incorporan en los sujetos desde el momento en que entran a los grupos estudiantiles de pertenencia. De allí surge el ritual de iniciación en la práctica política juvenil, que expone cómo el cuerpo empieza a revestirse de otros símbolos a través de un proceso práctico y continuo entre las estructuras dominantes y las potencias limitadas. Precisamente esto último muestra las potencialidades de los sujetos para incorporar las (dis)posiciones corporales.

En la indagación se pudo observar — a través del trabajo de campo- cómo estas prácticas políticas se inscriben en el cuerpo, lo cual conduce a un segundo resultado: el capital cultural del cuerpo es el de sus prácticas. Esto es, se interpreta que el cuerpo va siendo en las prácticas en la medida en que los procesos de incorporación avanzan de forma concatenada, simultáneamente de manera colectiva e individual. Los cuerpos analizados son cuerpos que ya son, pero también que van siendo; que se transforman en la medida en que, frente a las acciones políticas, van incorporando nuevas significaciones en la práctica política universitaria. En otras palabras, el caso estudiado muestra cómo 
las y los jóvenes se vinculan identitariamente a los grupos, a la vez que estos van trasformando sus (dis)posiciones para constituir cuerpos para la política.

Por otra parte, la respuesta a la pregunta sobre cómo se manifiesta lo corporal en las prácticas políticas de las y los militantes universitarios puede interpretarse como un tercer resultado. En este sentido, se evidencia que el cuerpo se manifiesta a través de prácticas políticas que muestran específicamente las potencialidades de lo corporal, en relación con las acciones de los sujetos en la sociedad. Son formas instituyentes de relacionarse consigo mismo, con los otros y con el espacio en el contexto del activismo universitario. Dicho de otro modo, si históricamente se ha pensado el cuerpo como objeto de reproducción de las relaciones de poder institucionalizadas, es también a través de lo corporal que se despliega la producción de las potencialidades que permiten interpelar ese mismo orden institucionalizado. De allí que el cuerpo sea interpretado como una potencia limitada (Galak, 2015), lo que implica una particular relación ética, estética y política de/con lo corporal.

Siguiendo esta línea, en el contexto de la investigación esta condición de lo social incorporado como una potencia limitada es especialmente manifiesta por el hecho de tratarse de un estudio sobre la juventud, condición de distinción valorada socialmente como capital simbólico. ${ }^{10}$ La juventud como sinónimo de energía y vigorosidad, sumada a la idea de cuerpo joven como acepción de fortaleza y virilidad, confluyen en el trabajo de campo cuando se pondera la novedad como constitutiva de la práctica política. Pero, a su vez, la moratoria que supone la juventud implica para los militantes universitarios saberse sujetos políticos en formación, con la inocencia (en tanto falta de responsabilidad, pero también portando una cierta ingenuidad) oculta en las vestimentas de la rebeldía. El resultado son prácticas de subjetivación política que definen la militancia universitaria a través de hexis corporales, ropas, cortes de cabello, etcétera.

Precisamente, las dimensiones de lo joven y lo corporal permitieron, no solamente esbozar estas consideraciones sobre las prácticas políticas universitarias, sino que a la vez expusieron una limitación de la presente investigación: analizando los resultados, es

1o Es importante resaltar que este trabajo tuvo como eje principal la perspectiva corporal en relación con la práctica política, por lo que los resultados permitieron la compresión y reafirmación del sujeto joven comprendido, más allá de la mirada adulto-céntrica, visualizándolo como agente capaz de incidir en los procesos sociales. 
importante remarcar que no se observó la perspectiva de género"1 como cuestión de singular importancia, la cual hubiese mostrado las particularidades distintivas en las transformaciones sociales (corporales y políticas).

Para finalizar, deseamos explicitar que el acento de este trabajo radica en la importancia de reflexionar sobre la relación cuerpo/jóvenes y prácticas políticas, como camino para la comprensión del cuerpo configurado en estos escenarios. Comprensión que emerge de los procesos de participación e incorporación de lo social que contribuyen a la construcción de los debates en las sociedades. Siguiendo esta lógica, se postula la política como una práctica que interpela al cuerpo, que se dispone en una forma singular de ser y hacer en la política y que, en el caso estudiado, presenta a las y los jóvenes del movimiento estudiantil como manifestación de la configuración de cuerpos para la política. Es relevante reconocer la potencialidad que desde el cuerpo emerge para proponer nuevas disposiciones sociales, en las cuales las y los jóvenes aparecen desde sus acciones y discursos incorporados en espacios propios; desde sus cuerpos se mueven con la intención de generar alternativas diferentes a las propuestas por la sociedad. De allí que esta reflexión haya ampliado la interpretación de la política más allá del concepto tradicional, puesto que esta debe comprenderse como un proceso atinente a los sujetos y a los cuerpos; un campo de disputas en donde las y los jóvenes irrumpen como agentes en la sociedad.

\section{Referencias}

Alvarado, S. V., Patiño, J., \& Loaiza, J. (2012). Sujetos y subjetividades políticas: el caso del movimiento juvenil Álvaro Ulcué. Revista Latinoamericana de Ciencias Sociales, Niñez y Juventud, 10(2), 855-869.

Berger, P., \& Luckmann, T. (1968). La construcción social de la realidad. Amorrortu. Bourdieu, P. (1994). Notas provisionales sobre el cuerpo. En C. Wright, M. Foucault, M. Pollak, H. Marcuse, J. Habermas, N. Elias, \& R. Castel (Eds.), Materiales de sociología crítica (pp. 183-194). La Piqueta.

Bourdieu, P. (2007). El sentido práctico. Siglo XXI.

\footnotetext{
${ }^{11}$ Es necesario aclarar que en uno de los grupos de discusión realizados con la Organización Estudiantil Territorio Libre participaron mujeres, pero sus relatos quedaron confinados a la narrativa grupal. Además, las dos historias de vida fueron hechas con hombres jóvenes. Es aquí donde se encuentra la falencia.
} 
Buendía, A. (2014). Narrativas urbanas y jóvenes escolarizados en Popayán: comunicación y educación en las formas de narrar y habitar la ciudad [Tesis doctoral no publicada]. Universidad del Cauca-Rudecolombia.

Butler, J. (2002). Cuerpos que importan. Paidós.

Butler, J. (2017). Cuerpos aliados y lucha política. Paidós.

Díaz, A. (2012). Devenir subjetividad política: un punto de referencia sobre el sujeto político [Tesis doctoral no publicada]. Centro de Estudios Avanzados Niñez y Juventud; Universidad de Manizales.

Díaz, A., \& Alvarado, S. V. (2012). Subjetividad política encorpada. Revista Colombiana de Educación, (63), 111-128. https://doi.org/10.17227/01203916.1689

Diz, C. (2018). Tácticas del cuerpo: activismo y resistencia en la ciudad en crisis. Disparidades. Revista de Antropología, 73(1), 127-152. https://doi.org/10.3989/rdtp.2018.01.005

Dukuen, J. (2018). Socialización política juvenil en un colegio de clases altas (Buenos Aires, Argentina). Revista Latinoamericana de Ciencias Sociales, Ninez y Juventud, 16(2), 867-880. https://doi.org/10.1160o/1692715x.16215

Enguix, B. (2012). Cuerpos y protesta: estrategias corporales en la acción colectiva. Revista Brasileira de Sociología da Emoção, 11(33), 885-913.

Figueroa-Grenett, C. (2018). La acción política de niños, niñas y jóvenes en Chile: cuerpos, performatividad y producción de subjetividad. Revista Latinoamericana de Ciencias Sociales, Ninez y Juventud, 16(1), 199-212. https://doi.org/10.11600/1692715x.16111

Font, R. J. (2015). Jóvenes y periferia. Universitat Oberta de Catalunya.

Galak, E. L. (2010). El concepto cuerpo en Pierre Bourdieu: un análisis de sus usos, sus límites y sus potencialidades [Tesis de maestría, Universidad Nacional de La Plata]. Repositorio Institucional de la UNPL. https://doi.org/10.35537/10915/52020

Galak, E. L. (2012). Laberintos de significado: cultura universitaria, jóvenes y cuerpos: un ensayo sobre definiciones conceptuales y subjetivación política. En A. Cervio (Comp.), Las tramas del sentir: ensayos desde una sociología de los cuerpos y las emociones (pp. 19-50). Estudios Sociológicos.

Galak, E. L. (2015). Esbozos de una teoría de la práctica de educar: Pierre Bourdieu, educación de los cuerpos, violencia y capital simbólico. Revista Tempos e Espaços em Educação, 8(15), 133-144. http://dx.doi.org/10.20952/revtee.v8115.3669

Galeano, M. (2004). Diseño de proyectos en la investigación cualitativa. Fondo Editorial Universidad Eafit. 
Giraldo, Y., \& Ruiz, A. (2019). Significados y alcances de la acción solidaria en jóvenes de Medellín. Revista Latinoamericana de Ciencias Sociales, Niñez y Juventud, 17(1), 301-314. https://doi.org/10.1160o/1692715x.17118

Hurtado, D. (2011). Entramados: jóvenes y configuración de significaciones imaginarias de deseo. Rudecolombia.

Le Breton, D. (2002). Antropología del cuerpo y modernidad. Nueva Visión.

Mauss, M. (1979). Sociología y antropología. Tecnos.

Pabón, A., \& Hurtado, D. (2016). «Mi piel es un lienzo»: sentidos de la modificación corporal en jóvenes de la ciudad de Cali. Revista Latinoamericana de Ciencias Sociales, Niñez y Juventud, 14(1), 477-491. https://doi.org/10.1160o/1692715x.14132150415

Patiño, J., Alvarado, S. V., \& Ospina-Alvarado, M. C. (2014). Ampliación de sentidos sobre las prácticas políticas de jóvenes con vinculación a siete movimientos sociales en Colombia. Revista Latinoamericana de Ciencias Sociales, Niñez y Juventud, 12(1), 257-275. https://doi.org/10.1160o/1692715x.12115101012

Reguillo, R. (2013). Culturas juveniles: formas políticas del desencanto. Siglo XXI.

Strauss, A., \& Corbin, J. (2002). Bases de la investigación cualitativa: técnicas y procedimientos para desarrollar la teoría fundamentada. Contus; Editorial Universidad de Antioquia. Universidad del Cauca. (2019). Unicauca en cifras. http://www.unicauca.edu.co/versionP/ node $/ 20070$ 